\title{
Strong Coupling Cavity QED with Gate-Defined Double Quantum Dots Enabled by a High Impedance Resonator
}

\author{
A. Stockklauser, ${ }^{*}$ P. Scarlino, ${ }^{\dagger}$ J. V. Koski, S. Gasparinetti, C. K. Andersen, C. Reichl, W. Wegscheider, \\ T. Ihn, K. Ensslin, and A. Wallraff \\ Department of Physics, ETH Zurich, CH-8093 Zurich, Switzerland \\ (Received 16 January 2017; revised manuscript received 17 February 2017; published 9 March 2017)
}

\begin{abstract}
The strong coupling limit of cavity quantum electrodynamics (QED) implies the capability of a matterlike quantum system to coherently transform an individual excitation into a single photon within a resonant structure. This not only enables essential processes required for quantum information processing but also allows for fundamental studies of matter-light interaction. In this work, we demonstrate strong coupling between the charge degree of freedom in a gate-defined GaAs double quantum dot (DQD) and a frequency-tunable high impedance resonator realized using an array of superconducting quantum interference devices. In the resonant regime, we resolve the vacuum Rabi mode splitting of size $2 g / 2 \pi=$ $238 \mathrm{MHz}$ at a resonator linewidth $\kappa / 2 \pi=12 \mathrm{MHz}$ and a DQD charge qubit decoherence rate of $\gamma_{2} / 2 \pi=$ $40 \mathrm{MHz}$ extracted independently from microwave spectroscopy in the dispersive regime. Our measurements indicate a viable path towards using circuit-based cavity QED for quantum information processing in semiconductor nanostructures.
\end{abstract}

DOI: 10.1103/PhysRevX.7.011030

Subject Areas: Mesoscopics, Quantum Physics

In the strong coupling limit, cavity QED realizes the coherent exchange of a single quantum of energy between a nonlinear quantum system with two or more energy levels, e.g., a qubit, and a single mode of a high quality cavity capable of storing individual photons [1]. The distinguishing feature of strong coupling is a coherent coupling rate $g$, determined by the product of the dipole moment of the multilevel system and the vacuum field of the cavity, which exceeds both the cavity mode linewidth $\kappa$, determining the photon lifetime, and the qubit linewidth $\gamma_{2}=\gamma_{1} / 2+\gamma_{\varphi}$, set by its energy relaxation and pure dephasing rates, $\gamma_{1}$ and $\gamma_{\varphi}$, respectively.

The strong coupling limit of cavity QED has been reached with a multitude of physical systems including alkali atoms [2], Rydberg atoms [3], superconducting circuits [4,5], and optical transitions in semiconductor quantum dots $[6,7]$. Of particular interest is the use of this concept in quantum information processing with superconducting circuits, where it is known as circuit QED [4,8,9].

Motivated by the ability to suppress the spontaneous emission of qubits beyond the free space limit [10], to perform quantum nondemolition (QND) qubit read-out

\footnotetext{
*anna.stockklauser@phys.ethz.ch

†pasquale.scarlino@phys.ethz.ch

*andreas.wallraff@phys.ethz.ch
}

Published by the American Physical Society under the terms of the Creative Commons Attribution 4.0 International license. Further distribution of this work must maintain attribution to the author(s) and the published article's title, journal citation, and DOI.
$[11,12]$, to couple distant qubits through microwave photons coherently $[13,14]$, and to convert quantum information stored in stationary qubits to photons $[15,16]$, research towards reaching the strong coupling limit of cavity QED is pursued for the charge and spin degrees of freedom in semiconductor nanostructures [17-22]. Recently, in parallel with the work discussed here, independent efforts to reach this goal have come to fruition with gate-defined DQDs in silicon [23] and carbon nanotubes [24].

The essence of our approach to reach the strong coupling limit with individual electronic charges in GaAs DQDs is rooted in the enhancement of the electric component of the vacuum fluctuations $\propto \sqrt{Z_{r}}$ [25] by increasing the resonator impedance $Z_{r}$ beyond the typical $50 \Omega$ of a standard coplanar waveguide. We have realized a frequency-tunable microwave resonator with impedance $Z_{r}=\sqrt{L_{r} / C_{r}} \sim$ $1.8 \mathrm{k} \Omega$ using the large inductance $L_{r} \sim 50 \mathrm{nH}$ of a SQUID array [26-28] combined with a small stray capacitance $C_{r} \sim 15 \mathrm{fF}$. Its resonance frequency, and thus also its impedance, is tunable by applying a small magnetic field using a mm-sized coil mounted on the sample holder. The frequency tunability of the resonator is particularly useful in this context, as it allows for the systematic study of its interaction with semiconductor nanostructures without changing their electrical bias conditions.

The resonator, with a small footprint of $300 \times 120 \mu \mathrm{m}^{2}$ [Figs. 1(a) and 1(b)], is fabricated using standard electronbeam lithography and shadow evaporation of aluminum (Al) onto a GaAs heterostructure. The embedded twodimensional electron gas (2DEG) has been etched away everywhere but in a small mesa region hosting the DQD. 

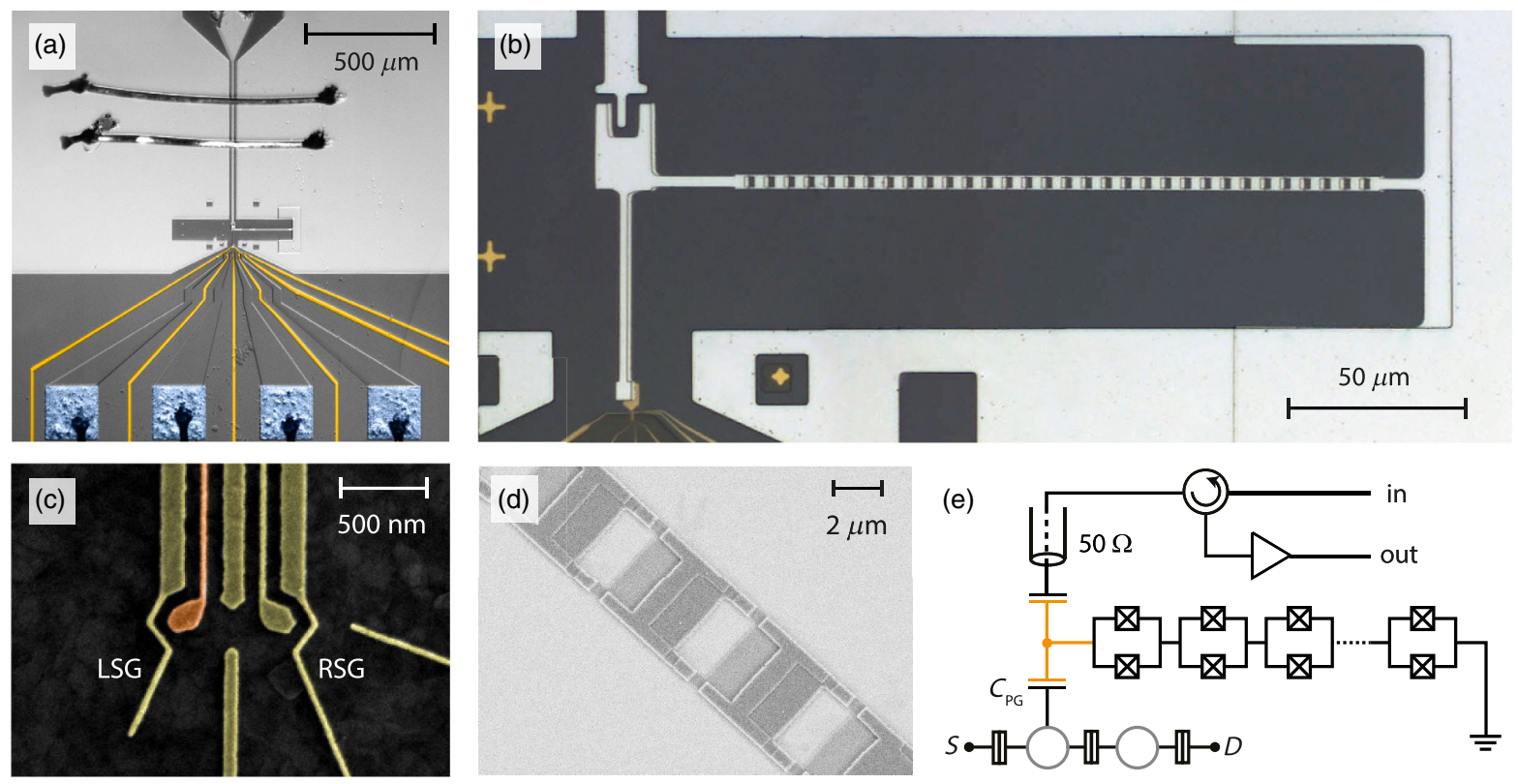

FIG. 1. Sample and simplified circuit diagram. (a) False-color optical micrograph of a representative device indicating the substrate (dark gray), the superconducting structures (light gray), the gold top gates (yellow) forming the DQD, and its source and drain leads and contacts (blue). (b) Optical micrograph displaying a SQUID array resonator (light gray) and its coupling gate to the DQD and the DQD biasing structures (yellow). (c) Electron micrograph of the DQD showing its electrostatic top gates (yellow) and the plunger gate coupled to the resonator (orange). (d) Electron micrograph of three SQUID loops (dark grey) in the array deposited on the etched GaAs heterostructure (light gray). (e) Circuit diagram schematically displaying the DQD (source contact labeled S, drain contact labeled D, and coupling capacitance $C_{\mathrm{PG}}$ to the resonator) and essential components in the microwave detection chain (circulator, amplifier) used for performing reflectance measurements of the device. Boxes with crosses and rectangles indicate Josephson and normal tunnel junctions, respectively.

The array, composed of 32 SQUIDs [Fig. 1(d)], is grounded at one end and terminated in a small island at the other end to which a single coplanar drive line is capacitively coupled. A gate line extends from the island and forms one of the plunger gates of the double quantum dot (orange) [Fig. 1(c)].

The double quantum dot is formed in the mesa structure using gold $(\mathrm{Au})$ top gates [yellow in Figs. 1(a)-1(c)] controlling the tunnel coupling of the DQD to the source and drain leads (blue) as well as the interdot tunnel coupling $t$. The left and right side gates (LSG, RSG) control the on-site electrostatic energies of each of the two dots, while the plunger gates are not biased in the experiment. An additional gate and pair of leads can be configured as a quantum point contact for charge detection. The microwave response of the system is probed in reflection [Fig. 1(e)] using standard circuit QED heterodyne detection techniques $[4,18]$.

We show that the resonance frequency of the SQUID array resonator can be tuned from a maximum value of $\nu_{r} \sim 6.0 \mathrm{GHz}$ to well below $4.5 \mathrm{GHz}$ (which is the lower cutoff frequency of our detection electronics) in measurements of its reflectance $\left|S_{11}\left(\nu_{p}\right)\right|$ as a function of applied magnetic flux $\Phi_{\mathrm{m}}$ and probe frequency $\nu_{p}$ [Fig. 2(a)]. From these data, we extract the characteristic circuit parameters of the resonator and find that its impedance changes from
$Z_{r} \sim 1.3 \mathrm{k} \Omega$ to $1.8 \mathrm{k} \Omega$ in this frequency range. From measurements of the SQUID array resistance at room temperature, we estimate a critical current of about $210 \mathrm{nA}$ per SQUID. The microwave drives applied to the resonator in the experiments discussed here create photon occupations on the order of one or below, resulting in currents in the resonator far below the critical ones. In this regime, the device has a linear response. At higher drive strength, the nonlinearity of the device is observed and may be characterized in spectroscopic measurements [29]. With the DQD well detuned from the resonator biased at $\nu_{r}=5.02 \mathrm{GHz}$, we determine its internal loss rate, its external coupling rate to the input line, and the total linewidth $\left(\kappa_{\text {int }}, \kappa_{\text {ext }}, \kappa\right) /(2 \pi) \sim(10.0,2.3,12.3) \mathrm{MHz}$ [30].

We configure the double quantum dot and determine its characteristic properties by extracting the amplitude and phase change of a coherent tone reflected off the resonator at frequency $\nu_{p}$ using a measurement of the reflection coefficient $S_{11}\left(\nu_{p}\right)$ in response to changes of the potentials applied to the gate electrodes forming the double quantum dot. Using this by-now well-established technique [17-19], we record characteristic hexagonal charge stability diagrams [Fig. 2(b)] from which we extract the DQD charging energy of $580 \mathrm{GHz}$ and estimate the number of charges in each dot to be of the order of 10 electrons [18,31]. 
(a)

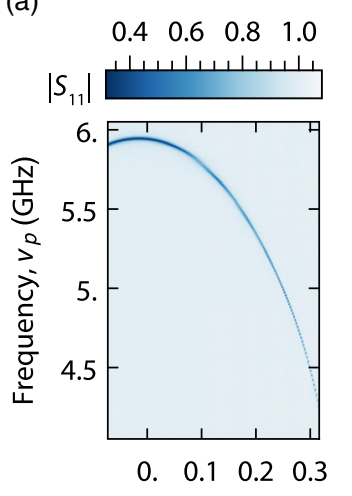

(b)

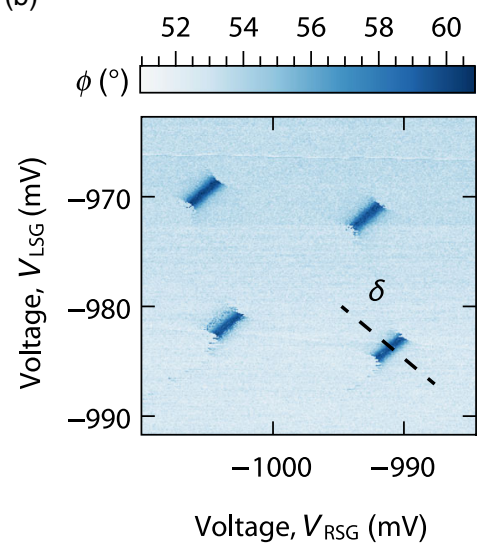

FIG. 2. Characterization of the SQUID array resonator and double quantum dot. (a) Reflectance spectrum $\left|S_{11}\right|$ of the resonator as a function of probe frequency $\nu_{p}$ and applied magnetic flux $\Phi_{\mathrm{m}} / \Phi_{0}$. (b) Hexagonal charge stability diagram of the DQD detected in the phase $\phi$ of the microwave tone at frequency $\nu_{p}$ reflected off the resonator close to its resonance frequency $\nu_{r}$ as a function of the applied side gate voltages $V_{\mathrm{RSG}}$ and $V_{\mathrm{LSG}}$.

To explore their mutual coupling, we first fix the SQUID array resonance frequency to $\nu_{r}=5.03 \mathrm{GHz}$ and set the tunnel coupling of the DQD to $2 t \sim 4.13 \mathrm{GHz}<\nu_{r}$. This ensures that tuning the difference energy $\delta$ between the charge states in the right and left quantum dots results in a resonance $\left(\nu_{q}=\nu_{r}\right)$ between the charge qubit transition frequency $\nu_{q}$ and the resonator at $\delta_{ \pm}= \pm \sqrt{\left(\nu_{r}\left(\Phi_{\mathrm{m}}\right)\right)^{2}-(2 t)^{2}}$.

Varying the detuning $\delta$ [along the dashed line indicated in Fig. 2(b)] by applying appropriately chosen voltages to the two side gates, we observe the dispersive (i.e., nonresonant) interaction between the DQD and the resonator in a probe-frequency-dependent reflectance measurement of the resonator [Fig. 3(a)]. As a function of $\delta$, the reflectance spectrum $\left|S_{11}\left(\nu_{p}\right)\right|$ shows characteristic shifts in the dispersive regime $\left(\nu_{q} \gg \nu_{r}\right.$ or $\left.\nu_{q} \ll \nu_{r}\right)$ and indications of an avoided crossing at $\delta_{ \pm} \sim \pm 2.86 \mathrm{GHz}$ at resonance $\left(\nu_{q}=\nu_{r}\right)$, which we analyze in more detail below.

We first extract the frequency $\tilde{\nu}_{r}$ of the resonator, as renormalized by its dispersive interaction with the DQD, by fitting a Lorentzian line to the reflectance spectrum at each value of $\delta$. The dispersive shift induced by the coupling to a qubit has previously been observed and used in circuit QED experiments with semiconductor double quantum dots [17-19]. When varying $\delta$, the experimentally extracted shift $\Delta \nu_{r}=\tilde{\nu}_{r}-\nu_{r}$ reaches up to about $100 \mathrm{MHz}$ close to resonance [blue dots, Fig. 3(b)]. The measured values of $\Delta \nu_{r}$ are in excellent agreement with the results of a master equation simulation (solid line) analyzed in the same way, finding the parameters $\left(g_{0}, \gamma_{1}^{b}, \gamma_{\varphi}^{b}\right) /(2 \pi)=(155,35,63) \mathrm{MHz}$ while keeping the bare resonator linewidth $\kappa$ fixed at its independently determined value stated above.

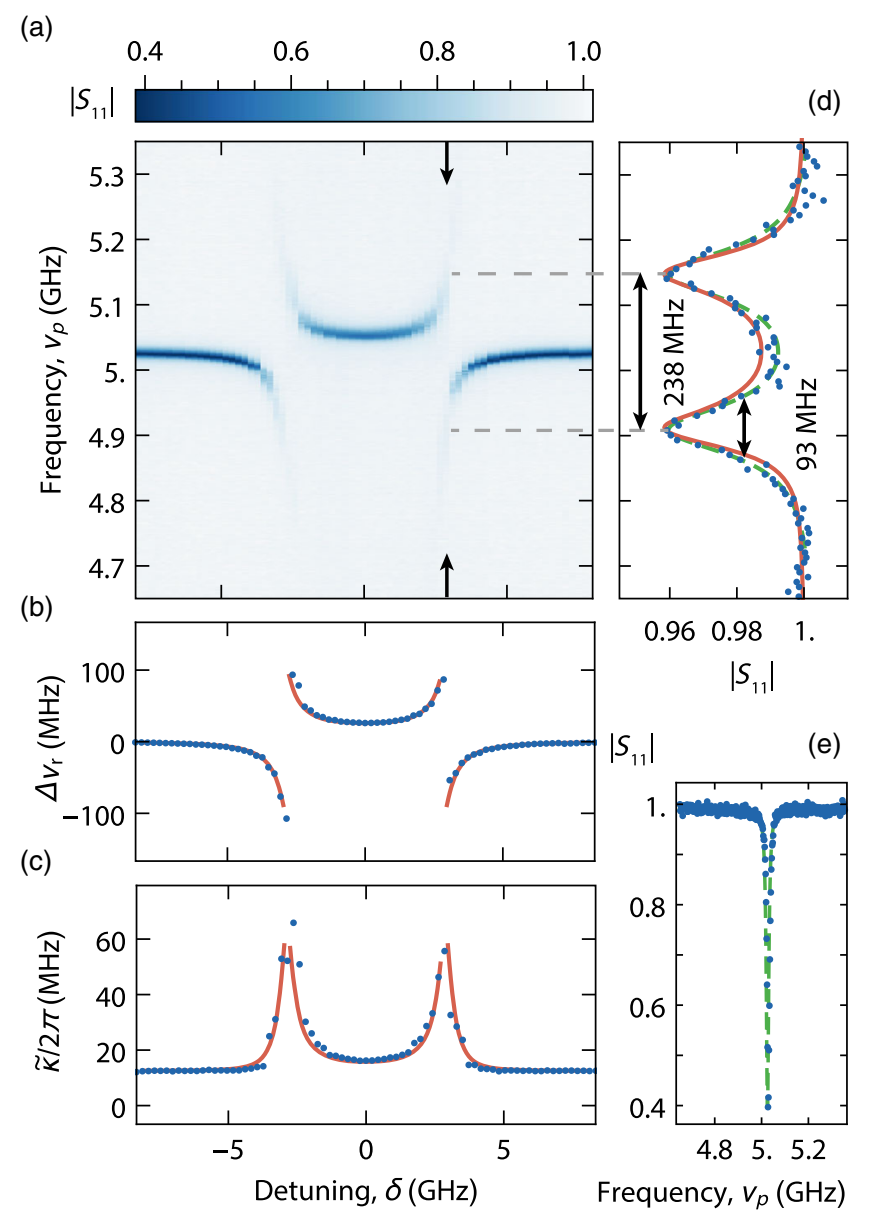

FIG. 3. Dispersive and strong resonant interactions. (a) Resonator reflectance $\left|S_{11}\right|$ as a function of probe frequency $\nu_{p}$ and DQD detuning $\delta$. The resonance $\left(\nu_{q}=\nu_{r}\right)$ occurring at $\delta_{ \pm}$is indicated by arrows. (b) Extracted resonator frequency shift $\Delta \nu_{r}$ (dots) and (c) linewidth $\tilde{\kappa}$ (dots) vs DQD detuning $\delta$ in comparison to results of a master equation simulation (line) for $\left(g_{0}, \gamma_{1}^{b}, \gamma_{\varphi}^{b}\right) /(2 \pi)=(155,35,63) \mathrm{MHz}$. (d) Measured resonator reflectance $\left|S_{11}\right|$ (dots) vs probe frequency $\nu_{p}$ at resonance $\left(\nu_{q}=\nu_{r}\right)$, displaying a strong-coupling vacuum Rabi mode splitting. The solid line is the result of the master equation simulation; the dashed line is a fit to a superposition of two Lorentzian lines. (e) Resonator reflectance spectrum $\left|S_{11}\right|$ with a Lorentzian fit (dashed line) in the dispersive regime vs probe frequency $\nu_{p}$.

In the Jaynes-Cummings model we use to describe the coupled system, both the coupling rate and the decoherence rates depend on the mixing angle $\theta$. The effective coupling strength $g$ is given by $g=g_{0} \sin \theta$, where $\sin \theta=2 t / \sqrt{(2 t)^{2}+\delta^{2}}$, while the decay and decoherence rates are given by $\gamma_{1}=\gamma_{\varphi}^{b} \sin ^{2} \theta+\gamma_{1}^{b} \cos ^{2} \theta$ and $\gamma_{\varphi}=\gamma_{\varphi}^{b} \cos ^{2} \theta+\gamma_{1}^{b} \sin ^{2} \theta$. This expression approximates the experimentally relevant energy relaxation and dephasing processes using a frequency-independent white-noise spectral density for both $\gamma_{\varphi}^{b}$ and $\gamma_{1}^{b}$. In the experiments presented here and the ones performed previously [18,31], 
we accurately model the observed linewidths within the experimentally explored frequency range using this approximation.

Using the set of parameters that models the dispersive frequency shift, we also find excellent agreement with the effective linewidth $\tilde{\kappa}$ of the resonator as renormalized by the hybridization with the DQD charge qubit. Detuned from the quantum dot, the resonator displays the bare linewidth $\kappa$. When approaching resonance, it is increased by more than a factor of 4 because of the interaction with the qubit with significantly larger linewidth $\gamma_{2} \gg \kappa$. Near resonance $\nu_{q} \sim \nu_{r}$, the resonator reflectance does not display a single Lorentzian line shape in probe frequency but develops two well-resolved spectral lines.

Tuning the DQD into resonance with the resonator $\left(\nu_{q}=\nu_{r}\right)$, indicated by arrows in Fig. 3(a), we observe a clear vacuum Rabi mode splitting (blue dots) in the reflectance spectrum of the resonator [Fig. 3(d)]. A fit (dashed green line) of the spectrum to a superposition of two Lorentzian lines yields a splitting of $2 g / 2 \pi \sim 238 \mathrm{MHz}$, with an effective linewidth of $93 \mathrm{MHz}$. The vacuum Rabi mode splitting is found to be in good agreement with the spectrum evaluated from the master equation simulation (red solid line) with the parameters $\left(g_{0}, \gamma_{1}^{b}, \gamma_{\varphi}^{b}\right) /(2 \pi)=$ $(155,35,63) \mathrm{MHz}$, which is consistent with the analysis of the dispersive frequency shift discussed above. We note that the small amplitude of the signal in reflection is a direct consequence of the fact that the qubit decoherence rate $\gamma_{2}$ is significantly larger than the resonator decay rate $\kappa$, an observation that is also reproduced in the theoretical analysis of the data.

Furthermore, we analyze the spectroscopic properties of the DQD charge qubit in two complementary measurements. First, we make use of the frequency tunability of the high impedance SQUID array resonator by applying a small magnetic flux $\Phi_{\mathrm{m}}$ to its SQUID loops and keeping the DQD charge qubit at a fixed tunnel coupling $2 t$. At a set of frequencies $\left\{\nu_{r}\left(\Phi_{m}\right)\right\}$, we observe resonator spectra characteristic for its dispersive and resonant interaction with the qubit [Fig. 4(a)]. The resonances $\left(\nu_{q}=\nu_{r}\right)$ occurring at $\delta_{ \pm}$ for the set of values $\left\{\nu_{r}\left(\Phi_{\mathrm{m}}\right)\right\}$ (red data points) are in good agreement with the expected dependence of the qubit energy levels on $\delta$ [see dashed line in Fig. 4(a)]. We note that at each resonance $\left[\nu_{q}=\nu_{r}\left(\Phi_{m}\right)\right]$ an avoided crossing displaying a vacuum Rabi mode splitting is observed.

We also perform qubit spectroscopy by probing the amplitude and phase of the resonator reflectance at fixed measurement frequency $\nu_{p}=5.947 \mathrm{GHz}$ while applying an additional spectroscopy microwave tone at frequency $\nu_{s}$ to the resonator. When the spectroscopy tone is resonant
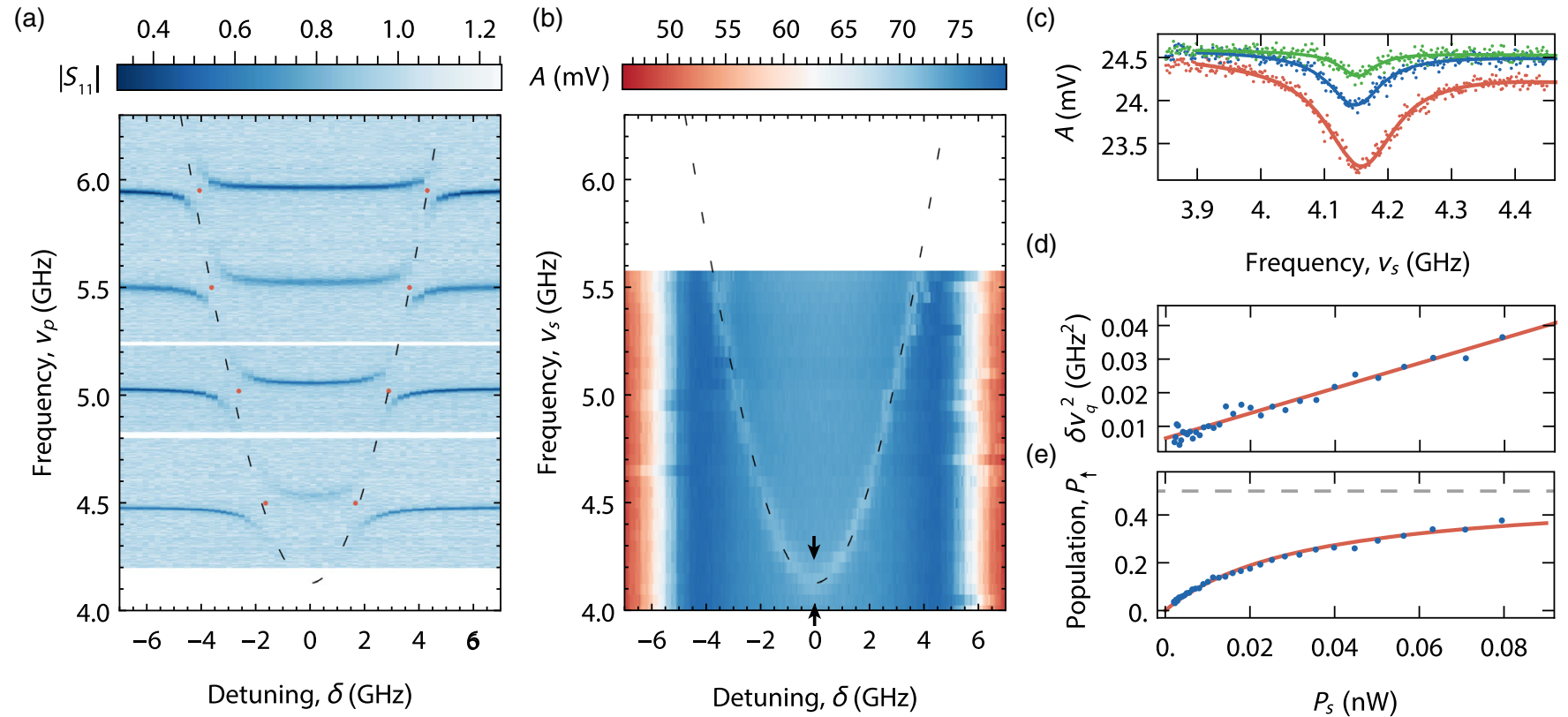

(e)

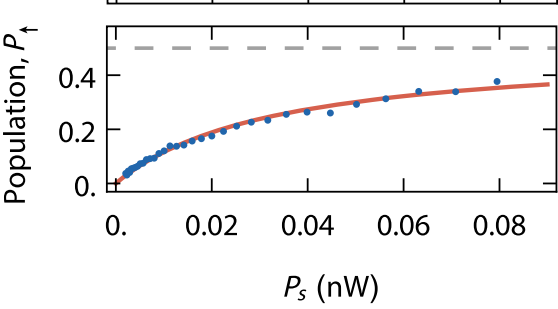

FIG. 4. DQD charge qubit spectroscopy. (a) Resonator reflectance spectra $\left|S_{11}\right|$ as a function of probe frequency $\nu_{p}$ and DQD detuning $\delta$ for a set of four independent measurements taken at $\nu_{r}\left(\Phi_{\mathrm{m}}\right) \approx\{4.5,5.0,5.5,5.9\} \mathrm{GHz}$. Red points indicate resonance $\left(\nu_{q}=\nu_{r}\right)$ extracted from the data. The dashed line indicates the calculated transition frequency of the charge qubit. (b) Amplitude $A$ of fixed frequency measurement tone $\nu_{p}=5.947 \mathrm{GHz}$ reflected from the resonator vs qubit spectroscopy frequency $\nu_{s}$ and qubit detuning $\delta$. The dashed line indicates the expected qubit resonance frequency for $2 t=4.13 \mathrm{GHz}$. (c) Qubit line shapes $A\left(\nu_{s}\right)$ (dots) measured at $\delta=0$ [arrows in (b)] for drive strengths $P_{s}=\{-5,-10,-15\} \mathrm{dBm}$ at the generator and fits to Lorentzian lines (on a linear background), extracting the full width at half maximum (FWHM) of the line $\delta \nu_{q}$. Probe frequency $\nu_{p}=5.022 \mathrm{GHz}$, and probe power $P_{p}=-35 \mathrm{dBm}$ at the generator. (d) Extracted qubit linewidth $\delta \nu_{q}^{2}$ (blue dots) vs spectroscopy drive power $P_{s}$ with linear fit (red solid line). (e) Saturation of qubit population with spectroscopy drive power $P_{s}$. 
with the qubit transition frequency $\left(\nu_{s}=\nu_{q}\right)$, the qubit is excited from its ground state $|g\rangle$ to a mixture between ground and excited states $|e\rangle$. This mixed state changes the resonance frequency $\tilde{\nu}_{r}$ of the resonator by dispersive coupling, resulting in a detectable change of the amplitude $A$ (and also of phase $\phi_{r}$, not shown) of the microwave tone reflected at frequency $\nu_{p}$ [Fig. 4(b)]. This technique has been pioneered for superconducting qubits [11,12], where it is widely used. Varying both the qubit detuning $\delta$ and the spectroscopy frequency $\nu_{s}$, we map out the spectrum of the qubit [dashed line, Fig. 4(b)] and determine its tunnel coupling $2 t=4.13 \mathrm{GHz}$.

Using this technique, we are not only able to accurately determine the transition frequency $\nu_{q}$ of the DQD charge qubit but also its line shape, shown for three drive powers $P_{s}$ in Fig. 4(c). The observed line shape depends on the qubit intrinsic linewidth, as set by its dephasing time $T_{2}^{\star}$, and on the strength of the applied microwave drive $P_{s}$, which broadens the line proportional to its amplitude. In the limit of weak driving $\left(P_{s} \rightarrow 0\right)$, the spectroscopic linewidth $\delta \nu_{q} \sim 80 \mathrm{MHz}$ (FWHM) is determined by the dephasing time $T_{2}^{\star} \sim 4.0 \mathrm{~ns}$ of the DQD qubit as extracted from a linear fit to the data in Fig. 4(d). This corresponds to a qubit decoherence rate $\gamma_{2} / 2 \pi=40 \mathrm{MHz}$. Increasing the drive strength $P_{s}$, we observe the qubit transition and thus also the resonator response to approach saturation [Fig. 4(e)].

Aspects in which the current device may still be optimized are the coherence properties of both the SQUID array resonator and the DQD. In characterization measurements of test samples, we found the observed internal loss rate $\kappa_{\text {int }} \sim 10 \mathrm{MHz}$ of the resonator to be dominated by residual coupling to the resistive and radiative loss channels provided by the normal metal leads of the DQD. In future device iterations, we plan to mitigate both effects by using superconducting $\mathrm{Al}$ gates and on-chip low pass filters [23,32]. Both measures may also improve the coherence properties of the DQD [23]. In previous measurements with coplanar waveguide resonators, we have not found a strong dependence of the DQD coherence on the number of charges down to the single electron regime [31]. In GaAs devices, the charge relaxation rate may ultimately be limited by the electron-phonon coupling in the piezoelectric host material [33]. This effect may be reduced by using materials such as silicon [34], which would also allow us to study the spin dynamics of individual electrons in an environment less prone to decoherence due to coupling to nuclear spins. To study spin dynamics at finite applied magnetic fields, it may also be interesting to explore alternative approaches to high impedance resonators such as the ones based on highkinetic-inductance thin-film resonators [35].

The data presented in this manuscript indicate that the strong coupling limit of a semiconductor charge qubit formed in a double quantum dot coupled to a microwave photon has been realized. This result is achieved by the use of a high impedance SQUID array resonator increasing the coupling strength by a factor of 6 relative to coupling schemes using conventional $50-\Omega$ resonators. This approach is universally applicable to any circuit QED application striving to maximize the coupling to the charge degree of freedom. The realization of strong coupling in this semiconductor circuit QED device also enabled us to perform spectroscopy of the DQD qubit in the dispersive regime to evaluate its line shape in dependence on the microwave drive power, indicating the possibility of temporally resolving the charge dynamics. These results carry promise to further advance quantum information processing efforts based on semiconductor charge and spin qubits using circuit QED approaches, e.g., to perform QND read-out and to realize coupling between distant qubits through microwave photons.

We acknowledge contributions by Michele Collodo, Andreas Landig, Ville Maisi, and Anton Potočnik. We thank Alexandre Blais for valuable feedback on the manuscript. This work was supported by the Swiss National Science Foundation through the National Center of Competence in Research (NCCR) Quantum Science and Technology and by Eidgenössische Technische Hochschule Zürich.

A. S. and P. S. contributed equally to this work.

[1] S. Haroche and J.-M. Raimond, Exploring the Quantum: Atoms, Cavities, and Photons (Oxford University Press, New York, 2006).

[2] R. J. Thompson, G. Rempe, and H. J. Kimble, Observation of Normal-Mode Splitting for an Atom in an Optical Cavity, Phys. Rev. Lett. 68, 1132 (1992).

[3] M. Brune, F. Schmidt-Kaler, A. Maali, J. Dreyer, E. Hagley, J. M. Raimond, and S. Haroche, Quantum Rabi Oscillation: A Direct Test of Field Quantization in a Cavity, Phys. Rev. Lett. 76, 1800 (1996).

[4] A. Wallraff, D. I. Schuster, A. Blais, L. Frunzio, R.-S. Huang, J. Majer, S. Kumar, S. M. Girvin, and R. J. Schoelkopf, Strong Coupling of a Single Photon to a Superconducting Qubit Using Circuit Quantum Electrodynamics, Nature (London) 431, 162 (2004).

[5] I. Chiorescu, P. Bertet, K. Semba, Y. Nakamura, C. J. P. M. Harmans, and J. E. Mooij, Coherent Dynamics of a Flux Qubit Coupled to a Harmonic Oscillator, Nature (London) 431, 159 (2004).

[6] T. Yoshie, A. Scherer, J. Hendrickson, G. Khitrova, H. M. Gibbs, G. Rupper, C. Ell, O. B. Shchekin, and D. G. Deppe, Vacuum Rabi Splitting with a Single Quantum Dot in a Photonic Crystal Nanocavity, Nature (London) 432, 200 (2004).

[7] J. P. Reithmaier, G. Sek, A. Loffler, C. Hofmann, S. Kuhn, S. Reitzenstein, L. V. Keldysh, V. D. Kulakovskii, T. L. Reinecke, and A. Forchel, Strong Coupling in a Single Quantum Dot-Semiconductor Microcavity System, Nature (London) 432, 197 (2004). 
[8] A. Blais, R.-S. Huang, A. Wallraff, S. M. Girvin, and R. J. Schoelkopf, Cavity Quantum Electrodynamics for Superconducting Electrical Circuits: An Architecture for Quantum Computation, Phys. Rev. A 69, 062320 (2004).

[9] R. J. Schoelkopf and S. M. Girvin, Wiring up Quantum Systems, Nature (London) 451, 664 (2008).

[10] A. A. Houck, J. A. Schreier, B. R. Johnson, J. M. Chow, Jens Koch, J. M. Gambetta, D. I. Schuster, L. Frunzio, M. H. Devoret, S. M. Girvin, and R. J. Schoelkopf, Controlling the Spontaneous Emission of a Superconducting Transmon Qubit, Phys. Rev. Lett. 101, 080502 (2008).

[11] D. I. Schuster, A. Wallraff, A. Blais, L. Frunzio, R.-S. Huang, J. Majer, S. M. Girvin, and R. J. Schoelkopf, AC Stark Shift and Dephasing of a Superconducting Qubit Strongly Coupled to a Cavity Field, Phys. Rev. Lett. 94, 123602 (2005).

[12] A. Wallraff, D. I. Schuster, A. Blais, L. Frunzio, J. Majer, S. M. Girvin, and R. J. Schoelkopf, Approaching Unit Visibility for Control of a Superconducting Qubit with Dispersive Readout, Phys. Rev. Lett. 95, 060501 (2005).

[13] J. Majer, J. M. Chow, J. M. Gambetta, J. Koch, B. R. Johnson, J. A. Schreier, L. Frunzio, D. I. Schuster, A. A. Houck, A. Wallraff et al., Coupling Superconducting Qubits via a Cavity Bus, Nature (London) 449, 443 (2007).

[14] M. A. Sillanpää, J. I. Park, and R. W. Simmonds, Coherent Quantum State Storage and Transfer Between Two Phase Qubits via a Resonant Cavity, Nature (London) 449, 438 (2007).

[15] A. A. Houck, D. I. Schuster, J. M. Gambetta, J. A. Schreier, B. R. Johnson, J. M. Chow, L. Frunzio, J. Majer, M. H. Devoret, S. M. Girvin, and R. J. Schoelkopf, Generating Single Microwave Photons in a Circuit, Nature (London) 449, 328 (2007).

[16] C. Eichler, C. Lang, J. M. Fink, J. Govenius, S. Filipp, and A. Wallraff, Observation of Entanglement Between Itinerant Microwave Photons and a Superconducting Qubit, Phys. Rev. Lett. 109, 240501 (2012).

[17] M. R. Delbecq, V. Schmitt, F. D. Parmentier, N. Roch, J. J. Viennot, G. Fève, B. Huard, C. Mora, A. Cottet, and T. Kontos, Coupling a Quantum Dot, Fermionic Leads, and a Microwave Cavity on a Chip, Phys. Rev. Lett. 107, 256804 (2011).

[18] T. Frey, P. J. Leek, M. Beck, A. Blais, T. Ihn, K. Ensslin, and A. Wallraff, Dipole Coupling of a Double Quantum Dot to a Microwave Resonator, Phys. Rev. Lett. 108, 046807 (2012).

[19] K. D. Petersson, L. W. McFaul, M. D. Schroer, M. Jung, J. M. Taylor, A. A. Houck, and J. R. Petta, Circuit Quantum Electrodynamics with a Spin Qubit, Nature (London) 490, 380 (2012).

[20] H. Toida, T. Nakajima, and S. Komiyama, Vacuum Rabi Splitting in a Semiconductor Circuit QED System, Phys. Rev. Lett. 110, 066802 (2013).

[21] A. Wallraff, A. Stockklauser, T. Ihn, J. R. Petta, and A. Blais, Comment on "Vacuum Rabi Splitting in a
Semiconductor Circuit QED System”, Phys. Rev. Lett. 111, 249701 (2013).

[22] J. J. Viennot, M. C. Dartiailh, A. Cottet, and T. Kontos, Coherent Coupling of a Single Spin to Microwave Cavity Photons, Science 349, 408 (2015).

[23] X. Mi, J. V. Cady, D. M. Zajac, P. W. Deelman, and J. R. Petta, Strong Coupling of a Single Electron in Silicon to a Microwave Photon, Science 355, 156 (2017).

[24] L. E. Bruhat, T. Cubaynes, J. J. Viennot, M. C. Dartiailh, M. M. Desjardins, A. Cottet, and T. Kontos, Strong Coupling Between an Electron in a Quantum Dot Circuit and a Photon in a Cavity, arXiv:1612.05214.

[25] M. H. Devoret, S. Girvin, and R. Schoelkopf, Circuit-QED: How Strong Can the Coupling Between a Josephson Junction Atom and a Transmission Line Resonator Be?, Ann. Phys. (Amsterdam) 16, 767 (2007).

[26] M. A. Castellanos-Beltran and K. W. Lehnert, Widely Tunable Parametric Amplifier Based on a Superconducting Quantum Interference Device Array Resonator, Appl. Phys. Lett. 91, 083509 (2007).

[27] N. A. Masluk, I. M. Pop, A. Kamal, Z. K. Minev, and M. H. Devoret, Microwave Characterization of Josephson Junction Arrays: Implementing a Low Loss Superinductance, Phys. Rev. Lett. 109, 137002 (2012).

[28] C. Altimiras, O. Parlavecchio, P. Joyez, D. Vion, P. Roche, D. Esteve, and F. Portier, Tunable Microwave Impedance Matching to a High Impedance Source Using a Josephson Metamaterial, Appl. Phys. Lett. 103, 212601 (2013).

[29] C. Eichler and A. Wallraff, Controlling the Dynamic Range of a Josephson Parametric Amplifier, Eur. Phys. J. Quantum Technology 1, 2 (2014).

[30] M. Göppl, A. Fragner, M. Baur, R. Bianchetti, S. Filipp, J. M. Fink, P. J. Leek, G. Puebla, L. Steffen, and A. Wallraff, Coplanar Waveguide Resonators for Circuit Quantum Electrodynamics, J. Appl. Phys. 104, 113904 (2008).

[31] J. Basset, D.-D. Jarausch, A. Stockklauser, T. Frey, C. Reichl, W. Wegscheider, T. M. Ihn, K. Ensslin, and A. Wallraff, Single-Electron Double Quantum Dot DipoleCoupled to a Single Photonic Mode, Phys. Rev. B 88, 125312 (2013).

[32] N. T. Bronn, Y. Liu, J. B. Hertzberg, A. D. Córcoles, A. A. Houck, J. M. Gambetta, and J. M. Chow, Broadband Filters for Abatement of Spontaneous Emission in Circuit Quantum Electrodynamics, Appl. Phys. Lett. 107, 172601 (2015).

[33] J. R. Petta, A. C. Johnson, C. M. Marcus, M. P. Hanson, and A. C. Gossard, Manipulation of a Single Charge in a Double Quantum Dot, Phys. Rev. Lett. 93, 186802 (2004).

[34] K. Wang, C. Payette, Y. Dovzhenko, P. W. Deelman, and J. R. Petta, Charge Relaxation in a Single-Electron $\mathrm{Si} / \mathrm{SiGe}$ Double Quantum Dot, Phys. Rev. Lett. 111, 046801 (2013).

[35] N. Samkharadze, A. Bruno, P. Scarlino, G. Zheng, D. P. DiVincenzo, L. DiCarlo, and L. M. K. Vandersypen, High-Kinetic-Inductance Superconducting Nanowire Resonators for Circuit QED in a Magnetic Field, Phys. Rev. Applied 5, 044004 (2016). 